De la Rosa Moreno, L. (2017). Objetos convertidos en sujetos: encuentros con voces excluidas dentro de una asignatura sobre inclusión educativa. Revista Electrónica Interuniversitaria de Formación del Profesorado, 20 (3), 209-223. DOI: http://dx.doi.org/10.6018/reifop.20.3.260111

\title{
Objetos convertidos en sujetos: encuentros con voces excluidas dentro de una asignatura sobre inclusión educativa
}

\author{
Lourdes de la Rosa Moreno
}

Profesora Contratada Doctora. Departamento de Didáctica y Organización Escolar, Facultad de Ciencias de la Educación, Universidad de Málaga.

\section{Resumen}

En este artículo se presenta y valora la forma de trabajar en la Asignatura de "Competencias Relacionales para el Aula Inclusiva", de la Mención "Escuela Inclusiva y Atención a la Diversidad", del Grado de Formación de Maestros/as en Educación Primaria de la Universidad de Málaga.

Su objetivo fundamental es la mejora de las competencias del alumnado universitario para la relación no discriminatoria con su futuro alumnado de Primaria (con un foco especial en aquellos pertenecientes a grupos no dominantes).

La metodología propuesta es la de "aprender haciendo", dentro de un contexto de aprendizaje dialógico en Grupos Interactivos (Comunidades de Aprendizaje).

Los resultados de la evaluación orientan sobre el alto grado de implicación requerido por quienes participan, pero también sobre la calidad de los procesos de aprendizaje, principalmente en los aspectos socio-afectivos.

\section{Palabras clave}

Educación Superior; Formación del profesorado basada en competencias; Educación Intergrupal; Educación Inclusiva. 


\title{
Objects become subjects: meetings with excluded voices in a subject about inclusive education
}

\begin{abstract}
In this article, the way of working in the subject "Relational Competences for the Inclusive Class", which belongs to Primary School Teachers' Formation Degree in University of Málaga, is presented and assessed.

The main goal is the improvement of the university students' competences in order to achieve a non-discriminatory relationship with their future primary students (with a special focus in those who are part of minority groups).

The proposed methodology is "learning by doing", in a dialogic Interactive Groups context (Learning Communities).

The evaluation outcomings shed light not only about the high implication required by those who participate, but also about the quality of learning procedures, mainly related with socio-affective aspects.
\end{abstract}

\section{Key words}

Higher Education; Competency Based Teacher Education; Intergroup Education; Minority Groups; Inclusive Education.

\section{Introducción}

El diseño de esta asignatura de "Competencias Relacionales para el Aula Inclusiva" (COMPREs) surge de la necesidad percibida por la autora, docente en la Universidad de Málaga desde 1996, de que el acercamiento que se suele ofrecer al alumnado de Magisterio hacia los niños y niñas de Educación Primaria sigue siendo, en gran medida, un acercamiento que, de algún modo, los "cosifica": los describimos, los clasificamos en grupos, enunciamos sus dificultades para las relaciones o para los aprendizajes académicos, ofrecemos programas y estrategias para la intervención con ellas y ellos...

Todo esto es necesario, pero el alumnado de Primaria vive, siente, aprende, llora y disfruta no siempre de la manera en la que aparece en los manuales psicopedagógicos (aun estando estos bien escritos y respondiendo con rigor, globalmente, a muchas de las realidades de las que en ellos se hable).

Por otra parte, tal y como afirman Gomilla, y Pascual (2015, p. 103), el análisis de la formación inicial del profesorado induce a "pensar en una invisibilidad de las familias como agente primordial en la educación de los niños y en una consideración del alumno de forma aislada de su entorno social concreto".

¿Por qué entendemos que es necesario, pero no suficiente estas formas de acercamiento a las alumnas y alumnos de Educación Primaria? Porque, como quien lee esto y como quien lo escribe, sean cuales sean las condiciones personales, familiares o socio-culturales, este alumnado no es objeto de docencia o estudio, sino sujeto de su propia vida en sus particulares contextos y, además, sujeto que convive con otros sujetos. 
Cuando, además, el alumnado no nos resulta familiar o cercano a nuestras propias experiencias o pertenecen a colectivos sobre los que es frecuente que se tengan prejuicios, visiones simplistas, trivializadas o tergiversadas de los mismos, aisladas de sus contextos, etc. (Torres, 2008), esta cosificación se acentúa y, por tanto, se agudiza la necesidad de vincularnos, de relacionarnos como sujetos irrepetibles, de rehacernos mutuamente desde el diálogo igualitario.

Este es pues el origen de esta asignatura de "Competencias Relacionales en el Aula Inclusiva" que, en los Planes de Estudio del Grado de Profesorado de Educación Primaria de la Universidad de Málaga (UMA) está incorporada en $4^{\circ}$ curso, dentro de la Mención de "Escuela Inclusiva y Atención a la Diversidad" (título redundante, útil solo para asuntos administrativos y de perfiles profesionales).

En la Guía docente de la asignatura aparece el Contexto en el que se enmarca: "El alumnado [en asignaturas previas] se ha introducido en el conocimiento, comprensión y toma de conciencia de que la cultura de la escuela inclusiva no es solo para aprender a darle respuestas a la diversidad en las aulas, sino para construir una escuela pública basada en el respeto a la diversidad como valor y no como defecto. En este sentido también está en juego nuestra propia reconstrucción personal. [... ] Desde esta asignatura, se pretende que el alumnado universitario desarrolle/ejercite sus propias Competencias Relacionales con el alumnado, familias y entornos socio-comunitarios menos afín a sus experiencias vitales, sociales, culturales, económicas, etc., intentando con ello alejarnos de perspectivas etnocéntricas y básicamente teorizantes sobre la atención a la diversidad en una escuela que se desea más inclusiva".

Se perfilan ya con lo dicho, los objetivos de la asignatura que pretenden acercar al alumnado a:

- Conocer, reconocer y valorar otros referentes culturales distintos al propio.

- Conocer, reconocer y valorar los propios referentes culturales.

- Reconocer las similitudes y diferencias intra e intergrupales.

- Asumir la existencia de situaciones de discriminación escolar que algunos colectivos vienen sufriendo en las escuelas y aulas (originadas tanto por personas como por contextos) e identificar sus causas para poder intervenir sobre ellas.

- Asumir que las propias COMP-REs pueden convertirse en una barrera o, por el contrario, en un facilitador de la inclusión en el aula.

- Ante realidades educativas complejas, como la atención a la diversidad en las aulas, ser capaz de percibir el valor de la riqueza que ofrece la comunicación y el trabajo compartido entre personas de los diversos colectivos implicados en la educación del alumnado (personas adultas pertenecientes a dicho colectivo, sus familiares, asociaciones, etc.).

- Desarrollar COMP-REs, desde el estudio y el contacto directo, para la comunicación y la docencia con el alumnado más vulnerable o en riesgo de exclusión.

Dichos objetivos concuerdan en lo esencial con los señalados por Chamseddine (2015, p. 76) para el ámbito escolar, a partir de los propuestos por Iglesias (2000): “... el objetivo del aprendizaje intercultural dentro del aula, es el desarrollo de la receptividad del alumnado ante las diferentes culturas, potenciando actitudes empáticas, asertivas, de respeto a la diversidad cultural, desde planteamientos reflexivos y críticos sobre la propia cultura a partir de otros marcos de referencia, considerando también las diferencias existentes en cada cultura, dadas por la edad, la clase social, el nivel económico, etc., generando a su vez identidades diferentes dentro de una misma cultura". 
Estas Competencias Relacionales se concretan y trabajan con contenidos tanto cognitivos (el conocimiento y la conciencia crítica, por parte del alumnado de la Mención y de la propia profesora, de elementos de la propia cultura y de otras, apuntes de la historia de los colectivos, etc.), como socio-emocionales (la detección y superación de los propios estereotipos y prejuicios, el control de la ansiedad, la capacidad de empatía, el interés hacia la comunicación entre diversas culturas, etc.) y, por supuesto, contenidos comportamentales o de acción (habilidades verbales y no verbales, flexibilidad comportamental, autoconfianza, etc.; en algún caso, habilidades relacionales específicas según colectivos).

La asignatura se ha desarrollado en los cursos 2013-14 y 2014-15, en un grupo de mañana (con más de 90 alumnas/os) y en otro de tarde (con, aproximadamente, 50).

Veamos, a continuación, el desarrollo metodológico que hemos seguido para alcanzar lo mejor posible, en el contexto comentado, las intenciones expresadas.

\section{Metodología}

Si aspiramos con metodologías tradicionales a que el alumnado desarrolle de manera integral sus competencias relacionales para el trabajo con su alumnado y las familias, probablemente pueda aprender fácilmente los contenidos teóricos o conceptuales, pero resultaría realmente difícil que hubiera cambios significativos en aspectos emocionales y en valores. Por ello, de acuerdo con De Miguel (2006), buscamos estrategias metodológicas vivenciales y prácticas, que incorporen experiencias de autoconocimiento y de desarrollo personal.

Como bien sintetizan las autoras Aróstegui y Darretxe (2016), para estas finalidades, se precisan cambios metodológicos referidos a los roles del alumnado: mucho más activo y participativo, tanto de manera individual como en grupos de trabajo, en el aprendizaje y en la evaluación; y de los docentes: con mayor protagonismo en la planificación, el seguimiento, etc., pero en un segundo plano durante las sesiones de clase, en un perfil más de "compañeros de viaje que ayudan al alumno a avanzar en la a veces necesaria deconstrucción y en la construcción o reconstrucción de su itinerario personal y profesional" (Barquín, Alzola y Madinabeita, 2012). Para unos y otros supone un cambio profundo respecto a lo que es enseñar y aprender.

Debo decir a continuación que, aunque la asignatura es mucho más, la actividad principal está inspirada en los Grupos Interactivos (GGII) propios de las Comunidades de Aprendizaje. Tal y como expresa Flecha (2009, p. 168), los grupos interactivos son:

... una forma de organización del aula contraria a la segregación que consiste en dividir el grupo clase en pequeños grupos de alumnos, lo más heterogéneos posible (en lo referente a niveles de aprendizaje, género, cultura, lengua, etcétera) y están dinamizados por personas adultas que no son profesionales de la educación, sino familiares de diferentes culturas, ex-alumnos, educadores de tiempo libre, personas de asociaciones y entidades del barrio, universitarios y universitarias, etcétera. Estas personas adultas voluntarias dinamizan y gestionan las interacciones en cada grupo, promoviendo la ayuda, la resolución conjunta de actividades a través del diálogo y el éxito de todas y todos.

En general, estos pequeños grupos heterogéneos trabajan desde el diálogo y son ayudados en sus actividades sucesivas y diferentes, durante un tiempo previsto, por dicho voluntariado adulto, igualmente heterogéneo, que participa como apoyo al desarrollo de las actividades propuestas en la asignatura. 
En nuestro caso, ¿quiénes son esas personas voluntarias con las que aprendemos? Solemos usar varios criterios a la hora de decidir los colectivos y sus representantes a los que invitamos a nuestras clases, como voluntariado con los que interaccionaremos. Menciono algunos de estos criterios sobre los que, de manera compleja, realizamos la selección:

- El primero es equilibrar la presencia de colectivos pertenecientes a los llamados "con diversidad funcional" (discapacidad) y a los llamados "con diversidad sociocultural" (en riesgo de desventaja social).

- Es relevante el que sean grupos que hayan tenido poca o ninguna presencia en el currículum real de este alumnado universitario previamente. Por ejemplo, el colectivo de personas con diversidad sexual y de género -LGTBI-.

- Personas en las que, frecuentemente, confluyen muchas necesidades de ajustes del entorno (físico, educativo, social...), como pueden ser las personas con parálisis cerebral.

- Grupos sobre los que han estudiado bastante y creen saberlo todo sobre "ellos", como son las personas con Síndrome de Down, las personas sordas o las que están en situaciones de inmigración.

- Por último, grupos ante los que tenemos especiales prejuicios "totalmente justificados desde el conocimiento que da el compartir el mismo territorio", como en tantos casos sucede con el pueblo gitano.

- La evaluación final que hace el alumnado universitario cada curso para la mejora de la asignatura.

El principio de procedimiento que nos guía, como decíamos, es ir pasando del aprender sobre ellos y ellas, al aprender con ellas y ellos, de verlos como objetos de estudio a relacionar-nos como sujetos, del explicar al escuchar-nos. $Y$ es ese proceso de escucha compartida el que puede llevar a la mejora de las COMP-REs ya que, como Cardoso-Belo (2012, p. 1302), pensamos que:

La mayoría de los modelos de competencia de comunicación se refiere a la competencia a nivel individual y la considera como un concepto que reside en el individuo. Otros modelos se centran en el sistema relacional, si bien no explican la variación individual o cultural. Buscamos que nuestro enfoque se centre en la relación entre los individuos, así como en los propios individuos, ocupándose de la dinámica de su interacción en las relaciones.

Las relaciones que propiciamos con estas personas que nos visitan en nuestras clases, como apuntábamos, se inspiran en los GGll que funcionan en las Comunidades de Aprendizaje, desde la perspectiva del aprendizaje dialógico que favorece la construcción de significados compartidos. Los procesos dialógicos implican que, más allá del imprescindible diálogo, estas interacciones estén basadas en el equilibrio de poder entre quienes participan, huyendo de considerar las diferentes aportaciones por las posiciones de poder de quienes las realizan (Flecha, 2001 y 2009). Es un principio ético que buscamos experimentar: el reconocimiento de que las experiencias y saberes de cada cual son imprescindibles, en este caso, para la construcción del conocimiento necesario para llegar a ser docente con COMPREs tanto con el alumnado perteneciente a los grupos dominantes o, simplemente, más afines al propio profesional, como con cualesquiera otros.

En el curso 2013-14 llevamos a cabo GGll con personas de 6 colectivos y, tras la evaluación final compartida con el alumnado, en el curso 2014-15, los redujimos a 4.

Así, invitamos a 6 personas voluntarias a participar en nuestras clases durante dos horas y media (cuatro sesiones en total en el curso 2014-15), para conversar con ellas y ellos. Esto supone un total de 24 visitas en cada uno de los grupos de la asignatura, el de mañana y el 
de tarde (insisto, concentradas en tan solo 4 sesiones) y, en tiempo, supone 10 horas de las 45 que tiene la asignatura.

La dinámica es la siguiente: 6 grupos de alumnado conversan sucesivamente, durante unos 20 minutos, con "las 6 visitas" de ese día, diseminadas en el aula de referencia del grupo y en algún otro espacio anexo.

Veamos una foto de una de las sesiones (fig. 1) y uno de los planos que se les entrega a nuestras visitas (que son quienes rotan en nuestra organización), con la secuencia que cada cual ha de seguir por los diferentes grupos (fig. 2).
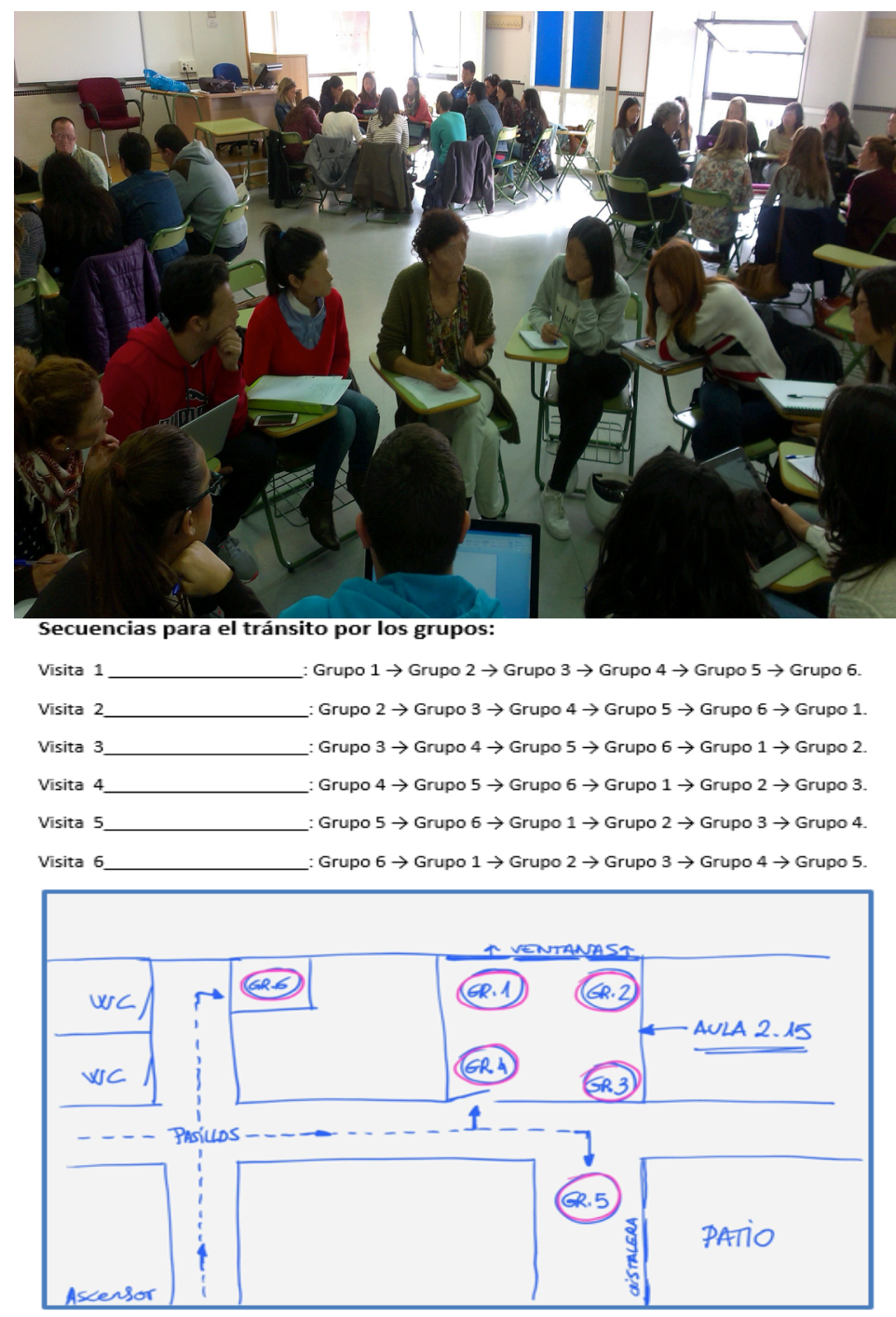

Fig. 1. Grupos interactivos en una sesión de mañana

Fig. 2. Plano y secuencia del tránsito por los grupos que ha de hacer cada visita de la tarde

Los perfiles de quienes vienen como voluntarios son los siguientes: dos personas pertenecientes a un determinado grupo cultural (etnia, capacidades personales, inmigración, género, clase social, orientación o identidad sexual,...); un familiar de las mismas (no tiene que ser de las presentes); un profesional no docente relacionado con el colectivo (investigador universitario, de la psicología y la pedagogía, del trabajo social, del ámbito sanitario,...); un maestro o maestra con experiencia y formación en la educación/relación con personas del colectivo del que se trate; $y$, por último, alguien del 
entorno comunitario (de alguna ONG relacionada, del vecindario, amistades, compañeros/as de estudios, comerciantes del barrio...).

Tras tener la experiencia directa en los GGIl, con una rúbrica orientativa (fig. 3), el alumnado elabora un Informe grupal sobre los aprendizajes realizados en cuanto a las COMP-REs que han desarrollado o necesitan considerar y mejorar en relación al alumnado perteneciente al colectivo concreto que sea.

\begin{tabular}{|c|c|}
\hline $\begin{array}{l}\text { Recogida y } \\
\text { selección de la } \\
\text { información }\end{array}$ & $\begin{array}{l}\text { La información que presenta es muy interesante. Aporta riqueza de } \\
\text { elementos de todos los componentes de las COMP-RE }\end{array}$ \\
\hline $\begin{array}{l}\text { Dominio del } \\
\text { contenido }\end{array}$ & $\begin{array}{c}\text { Muy buen dominio del contenido sobre COMP-RE y sobre el colectivo, } \\
\text { que emerge en el análisis, interpretación y conclusiones de la } \\
\text { información recogida }\end{array}$ \\
\hline $\begin{array}{l}\text { Mirada sobre el } \\
\text { colectivo }\end{array}$ & $\begin{array}{c}\text { Mantiene un buen equilibro en el acercamiento a la complejidad de lo } \\
\text { común entre los seres humanos, lo específico del grupo y lo particular de } \\
\text { cada individuo. } \\
\text { Supera el modelo del déficit }\end{array}$ \\
\hline Organización & $\begin{array}{c}\text { La organización resalta y focaliza excelentemente las ideas o temas } \\
\text { centrales. El orden, la estructura o la presentación comprometen y guían } \\
\text { a quien lee a lo largo del texto }\end{array}$ \\
\hline $\begin{array}{l}\text { Eficacia } \\
\text { comunicativa }\end{array}$ & $\begin{array}{l}\text { El escrito es muy interesante y enfocado al colectivo. Mantiene muy } \\
\text { bien la atención de quien lo lee. El tema central se enriquece con } \\
\text { anécdotas y detalles relevantes. Consigue que se den aprendizajes } \\
\text { significativos y que se conecte emocionalmente con el colectivo }\end{array}$ \\
\hline $\mathbf{V o z}$ & $\begin{array}{l}\text { Quienes escriben hablan directamente a quien lee en forma directa, } \\
\text { expresiva y comprometidos con el tema (se sienten implicados). Se } \\
\text { expresan interesantes reflexiones, cuestionamientos y aprendizajes } \\
\text { propios, bien fundamentados y coherentes con lo expuesto }\end{array}$ \\
\hline $\begin{array}{c}\text { Calidad de la } \\
\text { escritura }\end{array}$ & La escritura es completa y rica, además de concisa \\
\hline Convenciones & $\begin{array}{c}\text { Quienes escriben demuestran un excelente manejo de los estándares y } \\
\text { convenciones de la escritura } \mathrm{y}^{1} \text { y los usan muy exitosamente para facilitar } \\
\text { la lectura. Los errores tienden a ser muy pocos y de menor importancia, } \\
\text { al punto que el lector fácilmente puede pasarlos por alto, a menos que } \\
\text { los busque especificamente }\end{array}$ \\
\hline $\begin{array}{c}\text { Otras } \\
\text { observaciones }\end{array}$ & \\
\hline
\end{tabular}

Fig. 3. Rúbrica orientativa para los Informes: criterios y rango superior de desempeño

Una vez entregados los Informes, realizamos intercambios de los mismos entre los diversos grupos y cada Informe es coevaluado (sin calificación numérica, solo cualitativa), apoyándose en la rúbrica, por otros dos grupos, los cuales, de manera anónima entregan por escrito las principales fortalezas encontradas y algunas sugerencias de mejora.

Así, aunque la asignatura es mucho más, el grueso de las actividades gira alrededor de estas sesiones con los GGII.

Como actividades previas, por ejemplo, trabajamos sobre lo que son las COMP-REs y sobre fuentes de información que nos acercan a cada uno de los colectivos -preferentemente escritas o protagonizadas por personas pertenecientes a los mismos y siempre desde una perspectiva competencial y socio-cultural y no desde un modelo normativo, defectológico o médico-.

Algunas de las actividades posteriores a las sesiones de los GGIl son la mencionada elaboración grupal de los Informes y su coevaluación inter-grupos y, de manera destacada, la actividad final de la asignatura en la que deben aplicar lo vivido y aprendido, al diseño de un Taller de Formación en Competencias Relacionales (tras la deliberación con el alumnado, en el curso 2014-15 se rediseñó con mucha menor exigencia y complejidad, reduciéndose a la elaboración de material didáctico para dicha formación).

Este Taller está dirigido a sus compañeros y compañeras de clase (futuros docentes). Se inicia con el estudio teórico y el acercamiento real a algún colectivo poco visibilizado en la escuela o sobre el que entienden que necesitan acercarse desde una perspectiva y experiencia vivida más intercultural, más dialógica. El trabajo obliga pues al contacto 
directo con personas, asociaciones, etc. que pertenezcan o representen directamente al grupo en cuestión. Aparte de los sugeridos, se han realizado trabajos muy retadores, tanto para el alumnado que lo propone como para mí misma como docente, entre otros, por ejemplo, sobre diversidad familiar, con alumnado en situación de acoso por apariencia física, alumnado con madres prostitutas, alumnado en situación de acogida familiar o con alumnado transexual.

Realmente son espacios de desafío, ya que vuelve a evidenciarse la necesidad de reconstrucción personal del futuro profesorado para desarrollar relaciones igualitarias y no prejuiciosas también con las familias. Por ejemplo, respecto a este tema de la diversidad familiar, al alumnado le resulta muy difícil salir de su mirada reduccionista y negativa ante la misma, entendiendo esta diversidad inicialmente solo como "familias desestructuradas" con efectos perjudiciales para su progenie. Se refuerzan los resultados de los estudios de Gomilla, y Pascual (2015, p. 103):

Existe una fuerte tendencia a considerar la familia desde perspectivas de riesgovulnerabilidad social, o visiones que tienden a resaltar los aspectos negativos de su intervención en los procesos de socialización infravalorar o ignorar las capacidades de éstas para ofrecer un entorno educativo apropiado (al resaltar, por ejemplo, las dificultades que las transformaciones familiares han generado en el ejercicio de las funciones educativas en las familias) o bien el papel (negativo/negligente) de éstas en el control de otros agentes de socialización (medios de comunicación).

Aunque, finalmente, son capaces de ampliar su comprensión sobre lo que significa la diversidad familiar, de relacionarse con familias no convencionales con apertura para dejarse empapar de sus experiencias y revisarse en el proceso, de rescatar la realidad familiar diversa del propio grupo-clase universitario, de visibilizar el valor otorgado a sus propias familias no estándares por sus compañeras/os, de comprender la necesidad del diálogo más igualitario y no prejuicioso con las familias de su futuro alumnado de Primaria, de proyectar formas y maneras de facilitarlo en las escuelas, etc.

Pues bien, una vez elaborado dicho Taller, cada grupo lo pone a disposición de todo el grupo-clase y expone en clase lo más relevante del mismo (presentación que se evalúa y califica por procesos de coevaluación, también por medio de rúbricas que, por limitación de espacio, no podemos aportar). Los materiales didácticos producidos para la formación en COMP-REs (con procesos de evaluación y calificación complementaria de la profesora), adoptan formatos tan variados como presentaciones en Power Point o en Prezi, artículos, revistas digitales, blogs, folletos y pósteres, vídeos, pegatinas, etc.

El alumnado universitario, parafraseando al Profesor Santos Guerra, empieza a hacer visible la diversidad cotidiana existente en nuestras aulas y en las de Primaria (Santos, 1990), a transferir parte de lo aprendido a nuevas realidades y a percibirse a sí mismo como un factor que entorpece o facilita los procesos de inclusión en las escuelas.

\section{Resultados y conclusiones}

\section{La dificultad de encontrar el sentido de la asignatura}

En primer lugar, debo decir que inicialmente la asignatura provoca en el alumnado un alto grado de incertidumbre y no poca desconfianza. Una metodología nueva, la demanda de mucha participación cooperativa por parte del alumnado, la organización aleatoria de los grupos de trabajo, la falta de expectativas claras de lo que vamos a trabajar... 
En esta última cuestión, por ejemplo, cuesta semanas que la mayoría del alumnado cambie la perspectiva de que no hablamos de las COMP-REs que deben ir consiguiendo las niñas y niños de Primaria, sino que los destinatarios de nuestra acción somos nosotros y nosotras mismas.

El primer día de clase cuando Lourdes nos preguntó: "¿qué creéis que pueden ser las competencias relacionales?", no teníamos ni idea, e incluso yo pensaba que eran aquellas habilidades que había que desarrollar en los alumnos que presentaban una determinada condición. Ahora también sé que estas competencias nos competen a nosotros como futuros docentes [Alumna F, grupo B, curso 2013-14].

Mi evaluación de la asignatura ha ido cambiando desde el comienzo hasta el final, puesto que comencé con cierta incertidumbre, puesto que no entendía, en un inicio, el fin de dicha asignatura, el objetivo y cuál era nuestra función. Con el trascurso de las sesiones he aprendido muchísimo de la información documentada que se nos proporcionó antes del encuentro con cada colectivo y después, tras compartir aquellas charlas espontáneas, porque... [Alumno/a 8, grupo A, curso 2014-15].

Progresivamente, las partes del puzle inicial van cobrando sentido según iremos viendo.

\section{El valor dado a la manera de construir los aprendizajes}

Al concluir la asignatura es muy valorada esta manera de aprender, que se inicia en lo teórico, pero que toma sentido en las emociones, la participación y los contrastes que se experimentan en el encuentro con las personas que nos visitan en los GGIl:

Como planteamiento de asignatura, se ha llevado a cabo una arriesgada, original y exigente metodología, al querer trasladar y adaptar al ámbito universitario los grupos interactivos [Alumno A, curso 2013-14].

$Y$ por supuesto adquirir todos estos nuevos conceptos que no olvidaré por la forma en la que han entrado en mi cabeza junto con esa mezcla de sensaciones, emociones y aprendizajes de cada lunes. [Alumna G, grupo B, curso 2013-14].

[Nosotros] hemos decidido qué aprender mediante la formulación de preguntas [...]. Escuchar en primera persona los testimonios es algo que te hace reflexionar y sentir cosas, a veces, inexplicables. [Alumno/a H, grupo B, curso 2013-14].

Pienso que sin los grupos interactivos la asignatura no sería muy coherente, ya que trabajar solo lo teórico no basta para desarrollar realmente las COMP-REs [Alumno/a 14, grupo A, curso 2014-15].

Aparecen otras cuestiones metodológicas. Por ejemplo, la gran mayoría del alumnado valora también muy significativamente el contraste interno entre quienes participamos en la asignatura. Así, se percibe que los debates en gran grupo y las diversas actividades en pequeños grupos cooperativos redundan en ofrecer oportunidades para la mejora de las COMP-REs:

El trabajo cooperativo entre los pequeños grupos, a pesar de que es difícil ponerse de acuerdo, considero que es la mejor forma de ampliar nuestra visión [Alumno/a 7, grupo A, curso 2014-15].

Es necesario destacar, que todo este magnífico trabajo ha sido posible también porque todos y cada uno de los compañeros de la clase se han comportado y trabajado de una manera muy especial, en el que el compañerismo ha sido un todo, durante estos meses. Si queremos que una clase funcione necesitamos unos alumnos con ganas de trabajar, y esto ha quedado claramente demostrado [Alumna E, grupo B, curso 201314]. 
Igualmente, ven necesaria y valiosa, por la implicación y la libertad que les permite a la hora de orientar su aprendizaje, la participación activa por su parte y el trabajo previo a las sesiones de GGII (con la documentación previa de la que han de extraer las cuestiones sobre las que conversar):

Debo igualmente valorar que las visitas no fueron observadas bajo una mirada hueca del alumnado, sino que antes habíamos tenido la interiorización de lo que era la competencia comunicativa intercultural, algo que vimos al inicio del curso y que, de alguna forma, nos ponía en una posición específica. [Alumno D, grupo B, curso 201314].

El que hayamos tenido un papel activo con las visitas siendo los responsables de llevar adelante los grupos interactivos, nos ha servido para que se trabajen los materiales de la asignatura semana a semana [...]. [Alumna/o 6, Grupo B, curso 2014-15].

Definitivamente, la altísima valoración que hace todo el alumnado de la actividad sobre la que pivota el conjunto de la asignatura, los GGIl, es prácticamente unánime.

\section{El contenido que se ha apropiado el alumnado}

Acerquémonos a lo que reconocen como aprendizajes construidos o en proceso de reconstrucción. Aparecen recursos como conocimientos, habilidades, emociones, actitudes y valores, como un sistema complejo, personal, de percepción, de comprensión y de actuación. Recursos que se convierten en competencias al ser usadas para comprender y actuar ante problemas y situaciones nuevas (o que abordamos con nuevas miradas) de nuestra vida personal, social y profesional (Pérez Gómez, 2013).

Al hablar el alumnado de lo que ha aprendido en relación a sus Competencias Relacionales emergen elementos como: la empatía, la capacidad de autoconocimiento y autocrítica, la flexibilidad, la aceptación de la discrepancia y del disenso, el valor de la diversidad, las habilidades para el aprendizaje compartido, el compromiso personal con situaciones injustas, el reconocimiento de los peligros del etiquetaje, etc. Por su importancia, me extenderé en este apartado más que en ningún otro aunque, para hacerlo más breve, he construido un "collage" con los argumentos que aparecen en algunos de sus escritos de evaluación de la asignatura:

Esta asignatura me ha ayudado a abrir la mirada sobre distintos colectivos invisibilizados en la sociedad actual. Además, he logrado comprender que el "ellos" somos también "nosotros" en muchas ocasiones [Alumno/a 9, grupo A, curso 2014-15].

Los grupos interactivos han sido muy productivos y nos han ayudado a comprender a las personas, a tener empatía y a conocer aspectos de nosotros que no conocíamos [Alumna A, grupo B, curso 2013-14]. ... en un futuro quiero ser capaz de seguir desarrollando mi empatía y saber cómo tengo que llevar a cabo acciones y cómo las tengo que ejecutar sabiendo comprender a todos mis alumnos [Alumna $F$, grupo $B$, curso 2013-14].

Personalmente he conseguido eliminar algunos prejuicios y otros los voy a seguir trabajando para que desaparezcan [Alumno/a 12, grupo A, curso 2014-15]. Todo esto ha cambiado mi manera de ser y pensar sobre personas diferentes a mí, entendiendo que no podemos generalizar ni etiquetar [Alumno/a 13, grupo A, curso 2014-15].

Algunas de las personas que han venido han expuesto puntos de vista que no comparto para nada, pero estas cosas ayudan a que escuchemos y tratemos de empatizar con gente que opina cosas distintas pero que las argumenta. A mí, personalmente, me viene especialmente bien este "ejercicio" de control y comprensión [Alumna E, grupo B, curso 2013-14]. 
La asignatura me ha permitido revisarme desde el punto de vista de las competencias relacionales como futura docente, y tomar conciencia de cómo algunas actitudes pueden fomentar situaciones de injusticia, que obvian los Derechos Humanos con respecto a determinados colectivos [Alumno/a 1, grupo B, curso 2014-15].

De lo más relevante para mí es la transversalidad de los aprendizajes obtenidos en la asignatura (ha sido una experiencia para la vida) [Alumna D, grupo B, curso 2013-14].

Como nota final, me permito utilizar este último documento del curso, para referirme a una de las claves barajadas durante el desarrollo de la asignatura: la superación del modelo médico de la discapacidad. Es muy posible que algunos sectores sociales piensen que la solución a la diversidad funcional esté en el modelo médico, de la misma forma que en el Código Penal la solución a la diversidad social. Detrás de esto, seguramente, esté el juicio fenomenológico sobre lo visible y la ignorancia sobre las causas invisibles, especialmente en la diversidad social, lo que supone que en la diversidad social es probable que también impere un modelo patológico de la diversidad, consistente en una generalización epidémica a ciertos grupos y colectivos que, a falta de medicación, bueno es el Código Penal [...]. A lo mejor, después de habilitar el contexto, queda pendiente habilitar la perspectiva [Alumno D, grupo B, curso 2013-14].

La observación directa de las emociones y comportamientos del alumnado, así como que estos escritos podían ser anónimos, dan credibilidad a estas manifestaciones, aunque sin duda, en algún caso puede ser que ocurra lo que Díaz de Greñu y Anguita (2017) comentan al hablar de la evolución de los estereotipos del profesorado en torno al género y a la orientación sexual:

La diferencia con respecto a épocas pasadas estriba, según ponen de manifiesto las observaciones, en que se pone mayor empeño en disimular estas representaciones mentales porque las manifestaciones explícitas de sexismo y homofobia no se toleran socialmente. Se hacen mayores las diferencias entre los discursos formal e informal.

\section{El valor de la experiencia más allá del aula}

Por otro lado, la valoración de la experiencia por parte de las personas voluntarias que nos visitan, es también muy favorable.

En relación a esta afirmación, deseo comentar el hecho de que, cuando invito a participar en esta y otras actividades académicas a mujeres y hombres de diversas condiciones para que vengan a hablar en primera persona de su experiencia vital y educativa, encuentro muy diferentes actitudes ante la invitación. A aquellas que son activistas, militantes, defensoras de los derechos humanos, tanto en espacios públicos como privados, su recorrido les da firmeza en sus convicciones y seguridad en sus capacidades argumentativas. En algunas otras se percibe inseguridad e incredulidad, en diferentes grados, en las propias capacidades para la actividad propuesta. Sin embargo, progresivamente, estas emociones negativas también van transformándose en experiencias de valía personal y de empoderamiento. Refiriéndome a estos últimos casos, expresaba en otro escrito (De la Rosa, 2016, p. 168):

[...] las expresiones “¿seré capaz?”, “ipodré hacerlo?”, “¿qué interés puede tener mi vida?", "yo no sé dar clase", etc. no han sido respuestas inhabituales (aunque, por supuesto, otras muchas se sienten seguras y sabedoras del valor de sus aportaciones). Así que, al principio, se percibe entusiasmo, pero también inseguridad ante la propia capacidad de presentarse como protagonista ante un grupo de alumnas y alumnos universitarios y también se aprecian dudas de si lo que se tiene que "contar" 
despertará el interés suficiente. [...] Pero una vez que colaboran, ¿resultan capaces de ofrecernos su propia realidad reflexionada? La respuesta es rotundamente sí. Incluso aquellas personas que, por haber tenido escasa e inadecuada enseñanza, no han adquirido el desarrollo académico esperado en función de sus capacidades (De la Rosa, 2016, p. 168).

Entresaco algunos de los comentarios de nuestras visitas, que ratifican parte de las afirmaciones anteriores y aportan otras valoraciones:

Gracias a ti por darnos la oportunidad de que se escuchen nuestras voces (Orientadora Laboral de Fundación Secretariado Gitano).

Gracias por hacernos recordar en este día que todo vale la pena y sobre todo la suerte de haber conocido a personas tan maravillosas como son ellos y ellas [personas con Síndrome de Down] (Monitora de la Asociación Málaga Down).

[...] la sensación de apoyo y la magnífica terapia que fue lo que vivimos ese día no está pagado con nada. Nos quedamos con ganas de más, la sensación de que haya personas que quieran saber de cerca lo que es el Asperger y cómo nos sentimos los padres es algo maravilloso, te refuerza para seguir luchando cada día en esta carrera de fondo. [...] (Madre y Padre de un niño con Asperger).

Pasé un día estupendo conversando con tu alumnado [...], poder intercambiar opiniones con los jóvenes, de los que aprendí muchas cosas ese día [...]. (Orientadora educativa).

Quiero que les trasmita [al alumnado], que llené mis depósitos de fuerza, y eso es lo que me hace seguir en esta lucha absurda; son unos libres pensantes MARAVILLOSOS [...]. No sabes cuánto bien nos hace a las personas LGTB que se nos conceda un espacio como el que tú creaste el otro día para deshacer prejuicios y hablarle a personas que el día de mañana deberán educar a los niños y a las niñas en la igualdad y la no discriminación (Madre de una niña transexual).

Esta actividad es muy enriquecedora. No solo para vosotros [el alumnado], sino también para mí. Me ha servido para pararme en pensar en la situación que tengo. Que una va pasando el día y nunca se para a pensar en lo que está viviendo, en lo que está sintiendo (Hermanastra de un adulto con Síndrome de Down).

[... ] esta iniciativa, todavía es muy necesaria [para] la visibilizacion de la diversidad, para normalizar socialmente la realidad de las personas LGTB (Abogada y presidenta de la Asociación OJALÁ de Lesbianas, Gais, Bisexuales y Transexuales).

Mientras más participo en este tipo de actividades, más me estoy dando cuenta que puedo llegar a ser tan culto como cualquiera. Con las vivencias, oyendo libros o escritos [...]. Me ha encantado la experiencia y espero haberos acercado a mi idea de que mi parálisis no es "el" gran problema para aprender. El gran problema son las barreras educativas y sociales (Persona con parálisis cerebral).

\section{Críticas y sugerencias}

Algunas críticas y sugerencias de mejora al diseño y desarrollo de la asignatura emergen del análisis de las evaluaciones de la asignatura que hace el alumnado. También en este momento voy a seleccionar aquellos temas que son más relevantes para el objetivo de este texto.

Por un lado, un aspecto del contexto institucional. Me refiero al número de estudiantes por grupo, con una media de 50 alumnos en el de la tarde y unos 90 en el de la mañana, hacen que cualquier actividad de trabajo en pequeño grupo produzca un nivel de ruido que se 
convierte en una barrera para la comunicación; al mismo tiempo, los debates de gran grupo no permiten una participación equilibrada de todo el alumnado en el tiempo de una clase; es imposible asegurar la evaluación individual continua del estudiantado (cuando se es responsable, además de otras asignaturas simultáneamente); puesto que "solo" vienen 6 voluntarios/as en cada sesión, el número de alumnos/as en cada pequeño grupo es muy alto (de 8 a 15 miembros), lo que enriquece mucho, pero dificulta la organización interna de los mismos; etc.

El gran número de alumnado en la clase ha dificultado mucho este planteamiento. Como primera vez, ha resultado ser aceptable, aunque no ha salido todo lo bien que se podía esperar [Alumno A, curso 2013-14].

En los grupos interactivos, la comunicación ha sido a veces dificultosa por el ruido, ya que no podíamos entender a personas que tenían más dificultades a la hora de hablar [Alumna/o 3, Grupo B, curso 2014-15].

Por otro lado, es una valoración casi unánime el mucho trabajo que les supone la asignatura (a pesar de haberla "descargado" de contenidos y actividades muy sensiblemente del primer al segundo curso): estudio de textos escritos y vídeos, elaboración de temas o cuestiones para los GGll, producción de los informes, procesos de coevaluación, trabajo final de grupo..., siendo, además, gran parte del trabajo en grupo y, por otra parte, en un contexto de final de carrera y simultánea en el tiempo con la elaboración del Trabajo Fin de Grado.

El volumen de trabajo ha sido muy alto, teniendo en cuenta la situación del alumnado, que cursa último año de carrera, tiene varias asignaturas más y un trabajo de fin de grado que elaborar [Alumno B, curso 2013-14].

Sin embargo y a pesar de todo, tanto el alumnado, como el voluntariado y yo misma valoramos muy satisfactoriamente esta experiencia docente en la que nos embarcamos, y muchos errores y desajustes, parte del alumnado los ve con comprensión, dentro de un contexto de compromiso compartido y mejora constante.

Como a todos, me gustaría conocer otras realidades pero, dentro de lo posible, creo que las horas de la asignatura están exprimidas al máximo [Alumna/o 11, Grupo B, curso 2014-15].

En este aspecto "chapeau" por la organización. Esto hace que de cara al exterior pueda decir que esta asignatura ha sido la más elaborada desde dentro, la que mayor carga de trabajo ha implicado, la que más tiempo nos ha ocupado, la que más agobios nos ha supuesto al final de carrera, pero todo merece la pena por la gran y REAL experiencia de contacto con los colectivos que hemos tenido [Alumna/o C, curso 2013-14].

Por mi parte, el sentido que tiene esta asignatura me da suficiente motivación como para seguir superando los escollos analizados e ir haciendo una propuesta que, sin perder el sentido inicial de la misma, permita estar progresivamente más ajustada a las posibilidades reales de quienes participamos en ella.

\section{Para finalizar}

Para terminar el relato de esta experiencia, quiero hacer un reconocimiento público a aquellos alumnos y a aquellas alumnas que, durante la asignatura, se han ofrecido a hablar en primera persona en alguno de los GGll.

Usando de manera genérica la expresión "salir del armario", podemos decir que algunas de estas personas habían salido ya del armario en la Universidad, pero otras no. Su valentía al 
compartir desde la máxima dignidad sus realidades personales con el resto de la clase, me conmueve: dos jóvenes gais, una mujer lesbiana, un hombre y una muchacha gitanos, una joven que ha vivido en diversas familias de acogida, un joven transexual.

El clima social de estos grupos les ha permitido tener confianza, sentirse suficientemente seguros emocionalmente como para regalarnos generosamente sus experiencias de vida... contadas en primera persona.

También gracias a cada uno y a cada una de ellos y ellas, cada cual, como sujeto de nuestros aprendizajes, hemos ido construyendo conocimiento, temporal y revisable, pero propio.

\section{Referencias}

Aróstegui Barandica, I. y Darretxe Urrutxi, L. (2016). Estrategias metodológicas activas en la asignatura de "Bases de la Escuela Inclusiva" de la E.U. de Magisterio de Bilbao. REDU. Revista de Docencia Universitaria, 14(2), 315-340.

Barquín, A.; Alzola Maiztegi, N.; Madinabeita Medrano, M. (2012). Innovación curricular en los grados de maestro en la facultad HUHEZI (Mondragon Unibertsitatea): la materia Educación intercultural y educación en valores. REDU. Revista de Docencia Universitaria, 10(3) 171 -194.

De Miguel Díaz, Mario (2006). Modalidades de enseñanza centradas en el desarrollo de competencias. Orientaciones para promover el cambio metodológico en el Espacio europeo de educación superior. Oviedo: Ministerio de Educación y Ciencia y Universidad de Oviedo.

Cardoso-Belo, José Manuel (2012). Competencia comunicativa y nuevas fórmulas docentes: La emergencia de nuevos modelos de comunicación didáctica. Revista de Comunicación Vivat Academia, Año XIV, Nº Especial, 1291-1309.

Chamseddine, Mohamed (2015). La construcción de identidad compartida en un aula intercultural. Revista Electrónica Interuniversitaria de Formación del Profesorado, 18(3), 69-81. DOI: http://dx.doi.org/10.6018/reifop.18.3.238841

De la Rosa, Lourdes (2016). La voz de las personas con discapacidad en la formación inicial de docentes. International Journal of Educational Research and Innovation (IJERI), 5, 160-169.

Díaz de Greñu Domingo, S. y Anguita Martínez, R. (2017). Estereotipos del profesorado en torno al género y a la orientación sexual. Revista Electrónica Interuniversitaria de Formación del Profesorado, 20(1), 219-232. DOI: http://dx.doi.org/10.6018/reifop.20.1.228961

Flecha, Ramón (2001). Compartiendo palabras. Barcelona: Paidós.

Flecha, R. (2009). Cambio, inclusión y calidad en las comunidades de aprendizaje. Cultura y Educación, 2009, 21(2), 157-169.

Gomilla, $M^{a}$ Antonia y Pascual, Belén (2015). La participación de las familias en el sistema educativo: la percepción del profesorado en formación. Revista Electrónica Interuniversitaria de Formación del Profesorado, 18(3), 99-112. DOI: http://dx.doi.org/10.6018/reifop.18.3.199321

Iglesias, C. I. (2000). Diversidad cultural en el aula de E/LE: L interculturalidad como desafío y como provocación. Madrid: Espéculo. 
Pérez Gómez, Ángel Ignacio (2013). ¿Qué merece la pena aprender en la escuela de la era digital? Cuadernos de Pedagogía, 438, 74-79.

Santos Guerra, Miguel Ángel (1990). Hacer visible lo cotidiano. Teoría y práctica de la evaluación cualitativa de los centros escolares. Madrid: Akal Universitaria.

Torres Santomé, Xurxo (2008). Diversidad cultural y contenidos escolares. Revista de Educación, 345, págs. 83-110. 